\title{
Assistência de enfermagem a um paciente portador de pé diabético: um relato de experiência do estágio supervisionado
}

Nursing care for a patient with diabetic foot: an experience report of the supervised internship Atención de enfermería a un paciente con pie diabético: relato de experiencia de la pasantía supervisada

Janayra de Brito Parentes ORCID: https://orcid.org/0000-0001-6609-0254 Instituto de Ensino Superior Múltiplo, Brasil E-mail: janayrabp@gmail.com

Thaynara Mesquita do Nascimento ORCID: https://orcid.org/0000-0001-8064-4671 Instituto de Ensino Superior Múltiplo, Brasil E-mail: thaymesquita23@gmail.com

Gabriela Oliveira Parentes da Costa ORCID: https://orcid.org/0000-0001-9473-8986 Universidade Federal do Piauí, Brasil E-mail: gabiparents@hotmail.com

Giuliane Parentes Riedel

ORCID: https://orcid.org/0000-0002-0637-1094 Centro Universitário Santo Agostinho, Brasil E-mail: giulianeriedel@hotmail.com

Ricardo Clayton Silva Jansen ORCID: https://orcid.org/0000-0002-6392-8100

Universidade Federal do Maranhão, Brasil

E-mail: ricardojansen_20@hotmail.com

Roseane Débora Barbosa Soares

ORCID: https://orcid.org/0000-0002-3190-4868 Universidade Brasil, Brasil

E-mail: rosedebora@hotmail.com

Tatiana Custódio das Chagas Pires Galvão

ORCID: https://orcid.org/0000-0001-9907-0831

Faculdade Santo Agostinho, Brasil

E-mail: tatis.a@hotmail.com

Francileuza Ciríaco da Cruz

ORCID: https://orcid.org/0000-0003-2886-1817

Hospital Universitário da Universidade Federal do Piauí, Brasil

E-mail: francileuzaciriaco@ @otmail.com

Fábio Soares Lima Silva

ORCID: https://orcid.org/0000-0002-8795-3255

Fundação Municipal de Saúde, Brasil

E-mail: soares-fabio@hotmail.com

Ravena de Sousa Alencar Ferreira ORCID: https://orcid.org/0000-0001-7311-2212

Universidade Federal do Piauí, Brasil

E-mail: ravenaa89@gmail.com

Verônica Elis Araújo Rezende

ORCID: https://orcid.org/0000-0001-9076-3375

Universidade Federal do Piauí, Brasil

E-mail: veronicaelisrezende@yahoo.com.br

Yara Maria Rêgo Leite

ORCID: https://orcid.org/0000-0002-4868-2624

Hospital Universitário da Universidade Federal do Piauí, Brasil

E-mail: yara_leite189@hotmail.com

Felipe de Sousa Moreiras

ORCID: https://orcid.org/0000-0002-8703-1429

Universidade Federal do Piauí, Brasil

E-mail: felipe_moreiras007@ hotmail.com

Marília Silva Medeiros Fernandes ORCID: https://orcid.org/0000-0001-5819-1178

Centro Universitário Santo Agostinho, Brasil

E-mail: marilia_s_medeiros@hotmail.com 


\title{
Resumo
}

Introdução: O pé diabético configura uma das principais complicações do diabetes mellitus (DM). Esse termo é utilizado para caracterizar a presença de lesões na região dos pés ocasionadas pela neuropatia periférica, doença vascular periférica e deformidades. Metodologia: Trata-se de um estudo descritivo e observacional, do tipo estudo de caso. Realizado em um Hospital de Timon-MA, desenvolvido na disciplina de Estágio Curricular Supervisionado II, por acadêmicos de enfermagem, no período entre agosto e dezembro de 2018. Relato da Experiência: A prática de estágio em enfermagem requer um olhar sensível dos discentes e profissionais que os acompanham. Realizar o máximo de cuidados possíveis, contribui para que o aluno saia do estágio com experiências da realidade do cotidiano da profissão. Com esse pensamento, os autores aproveitaram a prática assistida para atender a um paciente do sexo masculino, com 63 anos, com diagnóstico de Diabetes Mellitus(DM) há 3 anos. O mesmo havia sido admitido proveniente de outro hospital da região para tratamento do pé diabético, lesão em Membro Inferior Direito(MID) com curativo oclusivo limpo e seco. Considerações finais: A atuação de Enfermagem descritas neste relato de experiência, não se restringem apenas ao paciente citado, mas, à todos os pacientes com pé diabético que necessitam de atendimento para tratamento da doença e suas complicações, e precisam de um acompanhamento individualizado e de qualidade.

Palavras-chave: Pé diabético; Cuidados de enfermagem; Ferimentos e lesões.

\begin{abstract}
Introduction: The diabetic foot is one of the main complications of diabetes mellitus (DM). This term is used to characterize the presence of lesions in the feet caused by peripheral neuropathy, peripheral vascular disease and deformities. Methodology: This is a descriptive and observational study, of the case study type. Held in a Hospital in Timon-MA, developed in the discipline of Supervised Curricular Internship II, by nursing students, in the period between August and December 2018. Experience report: The practice of nursing internship requires a sensitive view from students and professionals that accompany them. Carrying out as much care as possible, helps the student to leave the internship with experiences of the reality of the profession's daily life. With this in mind, the authors took advantage of assisted practice to care for a 63-year-old male patient diagnosed with Diabetes Mellitus (DM) 3 years ago. The same had been admitted from another hospital in the region for the treatment of diabetic foot, injury to the Right Lower Limb (MID) with a clean and dry occlusive dressing. Final considerations: The nursing activities described in this experience report are not restricted to the mentioned patient, but to all patients with diabetic foot who need assistance to treat the disease and its complications, and need individualized follow-up and care. quality.
\end{abstract}

Keywords: Diabetic foot; Nursing care; Wounds and injuries.

\section{Resumen}

Introducción: el pie diabético es una de las principales complicaciones de la diabetes mellitus (DM). Este término se utiliza para caracterizar la presencia de lesiones en los pies causadas por neuropatía periférica, enfermedad vascular periférica y deformidades. Metodología: Se trata de un estudio descriptivo y observacional, del tipo estudio de caso. Realizado en un Hospital de Timón-MA, desarrollado en la disciplina de Pasantía Curricular Supervisada II, por estudiantes de enfermería, en el período comprendido entre agosto y diciembre de 2018. Informe de experiencia: La práctica de la pasantía de enfermería requiere una mirada sensible de los estudiantes y profesionales que acompañan ellos. Llevar el mayor cuidado posible ayuda al alumno a salir de la práctica con experiencias de la realidad del día a día de la profesión. Con este pensamiento en mente, los autores aprovecharon la práctica asistida para atender a un paciente masculino de 63 años diagnosticado de Diabetes Mellitus (DM) hace 3 años. El mismo había sido ingresado desde otro hospital de la región para el tratamiento de pie diabético, lesión en Miembro Inferior Derecho (MID) con un apósito oclusivo limpio y seco. Consideraciones finales: El trabajo de enfermería descrito en este relato de experiencia, no se restringe al paciente mencionado, sino a todos los pacientes con pie diabético que necesitan asistencia para el tratamiento de la enfermedad y sus complicaciones, y necesitan un seguimiento y atención individualizados de calidad.

Palabras clave: Pie diabético; Cuidado de enfermera; Heridas y heridas.

\section{Introdução}

O pé diabético configura uma das principais complicações do diabetes mellitus (DM). Esse termo é utilizado para caracterizar a presença de lesões na região dos pés ocasionadas pela neuropatia periférica, doença vascular periférica e deformidades. Tais deformidades ocasionam o prolongamento das internações hospitalares e podem levar o paciente à morte. 
Elas levam na faixa de dez anos, após o diagnóstico, para aparecerem e geralmente estão associadas à alguma infecção, podendo evoluir para amputação do membro, se não forem tratadas precocemente (Cosson; Ney-Oliveira; Adan, 2015).

O acompanhamento do profissional de saúde à pessoa com diabetes é de grande importância, pois medidas inerentes da equipe de enfermagem como: orientações sobre o controle glicêmico, orientações referentes às complicações com os pés e a importância da aceitação de hábitos de vida mais saudáveis, realizam um plano de cuidado em conjunto com a pessoa, tornando as combinações necessárias e planejando as intervenções direcionadas (Vargas et al., 2017).

Para reduzir a quantidade de amputações, os enfermeiros devem conhecer sobre a estratificação do atendimento a estes pacientes, adotando medidas educativas com foco nos indivíduos com essa patologia específica e de acordo com sua distribuição sócio-demográfica (Santos et al., 2018). Desta forma, a ampliação do conhecimento sobre a patologia se torna importante, assim como os fatores determinantes para melhoria da qualidade de vida deste paciente.

Pelo exposto, o objetivo desse estudo é relatar experiência da assistência de estagiários de enfermagem, prestada a um paciente com pé diabético, com o intuito de propor discussões sobre a temática e ampliar o espaço para adoção de novas práticas e políticas que permitam a melhoria da atuação do enfermeiro e assistência aos usuários e a prevenção de complicações.

\section{Metodologia}

A abordagem metodológica executada configura um estudo qualitativo, descritivo do tipo relato de experiência. Para Daltro e Faria (2019) e Pereira et al. (2018) o estudo qualitativo valoriza a descrição, a interpretação e a compreensão de um dado coletado, sendo relevante para o entendimento de um objetivo obtido por meio da experiência de uma determinada realidade.

A vivência ocorreu em 2018, durante o Estágio Supervisionado de Enfermagem, em um hospital de pequeno porte na cidade de Timon - Maranhão, onde prestou-se assistência a um paciente do sexo masculino, com diagnóstico de Diabetes Mellitus. O enfoque utilizado foram os diagnósticos e intervenções de enfermagem.

Na oportunidade, para a execução do cuidado de enfermagem, aplicou-se o Processo de Enfermagem, nas seguintes etapas: Coleta de Dados; Diagnósticos de Enfermagem; Planejamento de Enfermagem; Intervenções e Avaliação da Assistência proporcionada, segundo a Resolução COFEN 358/2009 (Silva et al., 2013).

Para descrever a experiência, os pesquisadores foram guiados pelo Itinerário de Paulo Freire (Figura 1) (Heidemann et al., 2017). 
Figura 1: Itinerário de Paulo Freire.

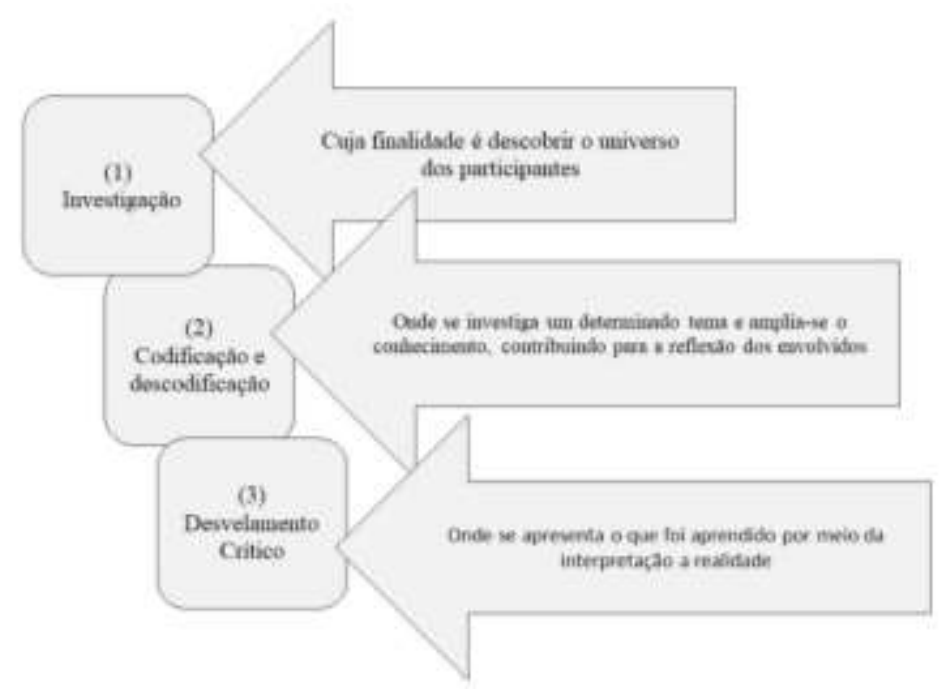

Fonte: Autores.

\section{Relato da Experiência}

A prática de estágio em enfermagem requer um olhar sensível dos discentes e profissionais que os acompanham. Realizar o máximo de cuidados possíveis, contribui para que o aluno saia do estágio com experiências da realidade do cotidiano da profissão.

Com esse pensamento, os autores aproveitaram a prática assistida para atender a um paciente do sexo masculino, com 63 anos, com diagnóstico de Diabetes Mellitus (DM) há 3 anos. O mesmo havia sido admitido proveniente de outro hospital da região para tratamento do pé diabético, lesão em Membro Inferior Direito (MID) com curativo oclusivo limpo e seco.

$\mathrm{Na}$ evolução de enfermagem, os discentes puderam entender um pouco mais sobre o paciente que estava no $9^{\circ} \mathrm{DIH}-$ dia de internação hospitalar para tratamento de lesões na região do pé, referenciada entre os profissionais da saúde como "Pé diabético". Ao exame físico, o paciente encontrava-se consciente, orientado, receptivo ao diálogo, mantendo pupilas isocóricas, mióticas e fotorreagentes. O mesmo estava respirando espontaneamente sem aporte de $\mathrm{O}^{2}$. Dieta seguida por via oral (VO), sem necessidade de auxílio e com boa aceitação. À ausculta pulmonar, mantinha murmúrios vesiculares presentes (+) e dispersos bilateralmente. Com relação à ausculta cardíaca, foi possível perceber bulhas normofonéticas, rítmicas e em 2T(2 tempos- TUM-TA; TUM-TA; TUM-TA). Com hemodinâmica estável. O abdômen estava globoso, flácido e sem sinais álgicos. Com temperatura registrando $36,5^{\circ} \mathrm{C}$. Sua pele estava normocorada e hidratada e mantinham acesso venoso periféricoAVP em membro superior direito (MSD). Diurese espontânea ausente no momento da avaliação. Evacuações espontâneas e ausente até o momento da avaliação.

Com relação a lesão evidenciada em MID, havia presença de esfacelo em quase toda sua extensão, necrose em um ponto específico de área pequena, tecido de granulação nas bordas, maceração e com exsudação moderada. Para o curativo foi usado alginato de cálcio e sódio, gazes, compressa estéril e atadura. O paciente referia queixas álgicas no membro lesionado.

Questionado sobre como estava sua alimentação em casa, o paciente relatou que não conseguia seguir a dieta prescrita pelo nutricionista. $\mathrm{O}$ mesmo relatou que não praticava nenhuma atividade física.

A partir dos achados pelos acadêmicos de enfermagem, foi possível estabelecer os diagnósticos relacionados abaixo (Figura 2). Todas os dados colhidos, assim como as intervenções planejadas, foram anotados no caderno dos estagiários e corrigidas pelo professor que os acompanhava. 
Figura 2: Diagnósticos de enfermagem prescritos, Maranhão, Brasil.

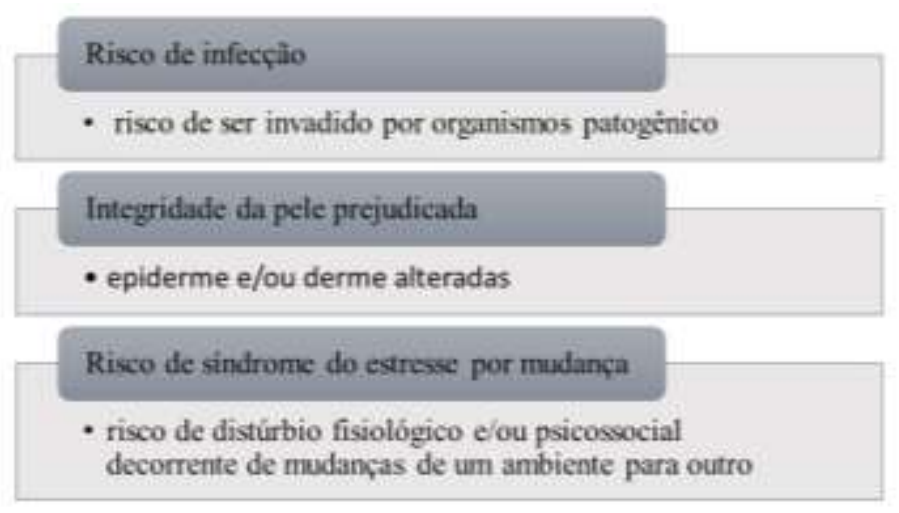

Fonte: Autores.

Assim, orientados pelo preceptor do estágio, os discentes elaboraram as seguintes prescrições de cuidados de enfermagem:

Explicar ao paciente sobre a importância da auto-monitorização e da monitorização e acompanhamento pelos principais profissionais de saúde.

> Orientar sobre a importância do uso de calçados e os devidos cuidados com os pés;

$>$ Orientar o paciente, sobre os cuidados com a pele, principalmente extremidades, sugerindo massagens com cremes ou soluções que amaciem a pele, aumentando a integridade da pele e diminuindo riscos de lesões;

$>$ Orientar sobre a importância das atividades físicas, e motivar a praticar caminhadas, enfim, exercícios físicos que não exijam recursos financeiros, se o mesmo não dispõe de recurso;

$>$ Reforçar a paciente sobre a importância de manter consultas de acompanhamento;

$>$ Aplicar o curativo apropriado para cicatrizar a lesão;

$>$ Administrar terapia com antibiótico;

> Monitorar os efeitos adversos das drogas;

> Monitorar o efeito terapêutico da antibioticoterapia na paciente.

Para o plano de alta, os discentes encorajaram o paciente fazer uso de um ambiente limpo e saudável, o esclareceram dúvidas sobre a higiene pessoal. Realizaram orientações sobre a continuidade dos medicamentos e a importância sobre o inspecionamento dos pés, com frequência. Além de incentiva-lo a uma dieta equilibrada, de acordo com sua patologia e a sempre monitorar os níveis de glicemia.

\section{Discussão}

Apesar de a DM estar relacionada a muitas complicações, as lesões na região dos pés apresentam maior incidência entre elas, sendo responsável por cerca de 70\% de amputações das extremidades inferiores (Fernandes et al., 2021).

A literatura evidencia que o número de homens diagnosticados com DM é maior que em mulheres, e considera essa probabilidade devido ao fato de que as mulheres cuidam mais da saúde (Caires \& Chiachio, 2020). De modo geral, é menos frequente a presença dos homens nas unidades de saúde. Assim, quando se trata de prevenção, eles não são figuras presentes, o que acarreta em maiores complicações relacionadas ao DM nesse gênero (Santos et al., 2020). 
Quando o acadêmico de enfermagem ou o profissional da equipe realiza estratégias voltadas para o cuidado, ele precisa sistematizar suas ações, visto que a SAE é uma ferramenta eficaz para proporcionar qualidade e a eficiência desse cuidado. Assim, a partir da evolução foi possível identificar os diagnósticos necessários para priorizar as intervenções.

O estagiário precisa entender que o profissional de enfermagem tem um papel importante no processo do cuidar, devendo aproveitar as oportunidades que as práticas oferecem para facilitar o reconhecimento dos riscos e complicações que afetam o paciente diabético. Assim, o exame físico, é fundamental para identificar quais os diagnósticos serão aplicados a cada paciente, individualmente.

Outra medida relevante é a orientação em saúde. Através das informações disponibilizadas ao paciente, no momento da alta, é possível prevenir ou melhorar o quadro de saúde deste, uma vez que a desinformação sobre os cuidados diários como o tipo ideal de calçado, a importância de secar os pés, a higiene e até mesmo a atividade física, podem contribuir com a melhora das complicações do indivíduo (Brandão, 2020).

\section{Considerações Finais}

O enfermeiro é uma peça fundamental para a prevenção de lesões nos membros inferiores e essencial para a educação dos pacientes que tenha diabetes.

A atuação de Enfermagem descritas neste relato de experiência, não se restringem apenas ao paciente citado, mas, a todos os pacientes com pé diabético que necessitam de atendimento para tratamento da doença e suas complicações, e precisam de um acompanhamento individualizado e de qualidade.

O estágio curricular no âmbito hospitalar tornou-se uma experiência gratificante, proporcionando aprendizagem, crescimento profissional, além de despertar o compromisso e encorajamento para a atuação na assistência de enfermagem, frente as diversas barreiras existentes nos serviços de saúde.

Assim, é fundamental que novos relatos de experiências vivenciadas durante a prática de enfermagem sejam publicados, a fim de fortalecer a cultura da assistência prestada pelos estagiários, desmistificando a ideia de que estes não possuem segurança para o cuidado, além de descrever a importância dessa etapa da graduação para os mesmos.

\section{Referências}

Brandão, M. G. S. A. (2020). Processo de enfermagem em paciente com pé diabético: relato de experiência. Rev. Rede cuid., Saúde, 14(1).

Caires, S. S. G., \& Chiachio, N. C. F. (2021). Prevalência de Hipertensão Arterial Sistêmica e Diabetes Mellitus entre os Trabalhadores da Indústria de Vitória da Conquista, Bahia. Id on Line Rev. Mult. Psic., 14(51).

Cosson, I. C., Ney-oliveira, F., \& Adan, L. F. (2005). Evalution of the knowledge of preventive measures for the diabetic foot in patients of, Acre. Arquivos Brasileiros de Endocrinologia \& Metabologia, 49(4).

Daltro, M. R., \& Faria, A. M. (2019). Relato de experiência: Uma narrativa científica na pós-modernidade. Estud. pesqui. psicol., 19 (1).

Fernandes, D. M. A. P. et al. (2021). A insustentável leveza do toque: reconhecimento do cuidado ao pé diabético. Saúde coletiva, 11(61).

Heidemann, I. T. S. B., et al. (2017) Reflections on Paulo Freire's research itinerary: contributions to health. Texto Contexto Enferm, 26(4).

Pereira, A. S., et al., (2018). Metodologia da Pesquisa Científica. UAB/NTE/UFSM.

Santos, L. C., et al. (2020). Envelhecimento, Tecnologia e Saúde: Uma Experiência Multidisciplinar para Promoção do Envelhecimento Saudável. XXVIII Ciclo De Palestras Sobre Novas Tecnologias Na Educação. At: Porto Alegre.

Vargas, C. P., et al. (2017). Condutas dos enfermeiros da atenção primária no cuidado a pessoas com pé diabético. Rev. enferm. UFPE on line, 11(11) p. 